\title{
Changes in preoperative and postoperative lipoprotein-associated phospholipase A2 activity in patients with malignant obstructive jaundice
}

\author{
Ying Zhao ${ }^{1,2}$, Guofang Feng ${ }^{3}$, Limin Feng ${ }^{1,2}$ \\ ${ }^{1}$ Department of Laboratory Medicine, The First Affiliated Hospital, College of Medicine, Zhejiang University, Hangzhou, China; ${ }^{2}$ Key Laboratory \\ of Clinical In Vitro Diagnostic Techniques of Zhejiang Province, Hangzhou, China; ${ }^{3}$ Department of Reproductive Endocrinology, Women's \\ Hospital, School of Medicine, Zhejiang University, Hangzhou, China \\ Contributions: (I) Conception and design: L Feng; (II) Administrative support: L Feng; (III) Provision of study materials or patients: Y Zhao; (IV) \\ Collection and assembly of data: Y Zhao, G Feng; (V) Data analysis and interpretation: Y Zhao; (VI) Manuscript writing: All authors; (VII) Final \\ approval of manuscript: All authors. \\ Correspondence to: Limin Feng. Department of Laboratory Medicine, The First Affiliated Hospital, College of Medicine, Zhejiang University, \\ Hangzhou 310003, China. Email: FLM@zju.edu.cn.
}

Background: Malignant obstructive jaundice (MOJ) leads to hyperbilirubinemia and systemic pathophysiological changes. The main clinical treatments for MOJ include radical pancreatoduodenectomy, palliative surgical treatment, and minimally invasive treatment, which can relieve biliary obstruction, drain bile, reduce jaundice, and improve liver function. In rat models, hepatic exposure to endotoxin resulted in rapid increases in biliary and plasma lipoprotein-associated phospholipase A2 (Lp-PLA2) levels, and our previous study revealed that Lp-PLA2 activity was strongly associated with liver damage. The present study aimed to clarify the serum Lp-PLA2 activity changes and evaluate the associations between Lp-PLA2 activity and laboratory parameters in MOJ patients preoperatively and postoperatively.

Methods: Twenty-one patients with MOJ were enrolled in this prospective study. Lp-PLA2 activity and other laboratory parameters were analyzed using a Hitachi 7600 automatic biochemical analyzer. Spearman correlation coefficients, percent differences, and dynamic difference plots were used to evaluate the changes in preoperative and postoperative Lp-PLA2 activity and the associations of Lp-PLA2 activity with other laboratory parameters.

Results: The postoperative Lp-PLA2 activity at 1 day [646 (range, 175-1,025) U/L], 1 week [419 (range, 144-949) U/L], and day of hospital discharge (347 (range, 165-698) U/L) differed significantly from the preoperative baseline activity [636 (range, 172-1,664) U/L; $\mathrm{P}<0.05$ for all]. Lp-PLA2 activity was correlated with total bilirubin $(\mathrm{TB})$ at specific time points $(\mathrm{P}<0.05$ for all). The percent differences and dynamic difference graphs revealed that Lp-PLA2 activity, alanine aminotransferase (ALT), aspartate aminotransferase (AST), and TB gradually decreased after biliary obstruction was relieved by surgical treatment.

Conclusions: Lp-PLA2 activity in MOJ patients was associated with biliary obstruction and liver damage. Serum Lp-PLA2 can be used as a novel indicator for biliary obstruction severity and treatment monitoring.

Keywords: Malignant obstructive jaundice (MOJ); lipoprotein-associated phospholipase A2 activity (Lp-PLA2 activity); dynamic difference graph

Submitted Jan 08, 2020. Accepted for publication Jul 05, 2020.

doi: $10.21037 /$ apm-20-133

View this article at: http://dx.doi.org/10.21037/apm-20-133 


\section{Introduction}

Malignant obstructive jaundice (MOJ) is frequently caused by malignant tumors, including primary carcinoma of the liver, carcinoma of the head of the pancreas, gallbladder cancer, cholangiocarcinoma, and periampullary tumor (1-5). MOJ leads to hyperbilirubinemia and systemic pathophysiological changes, such as gastric mucosal injury, liver and kidney failure, coagulation dysfunction, and immune hypofunction (2-5). The mechanism of the associated injury is complex, and involves endotoxemia, oxygen free radicals, cytokines, bile components, and other factors (6). Biliary obstruction can occur in any part of the biliary system (7). The diagnosis of obstructive jaundice mainly depends on clinical manifestations, biochemical marker detection, tumor marker detection, and imaging examinations [including ultrasound, computed tomography, magnetic resonance imaging, magnetic resonance cholangiopancreatography, and endoscopic retrograde cholangiopancreatography (ERCP)] (7-10). These imaging examinations are mainly used to determine the etiology, location, nature (benign/malignant), and degree of severity of the obstruction $(9,10)$. At present, the main clinical treatments for MOJ include radical pancreatoduodenectomy, palliative surgical treatment, and minimally invasive treatment [ERCP, endoscopic nasobiliary drainage (ENBD), endoscopic retrograde biliary drainage, endoscopic metal biliary endoprosthesis, and percutaneous transhepatic cholangiography and drainage (PTCD)] (7,11-14). These treatments can relieve biliary obstruction, drain bile, reduce jaundice, and improve liver function.

Lipoprotein-associated phospholipase A2 [Lp-PLA2; also known as platelet-activating factor acetylhydrolase (PAF-AH)] is mainly secreted by macrophages and catalyzes hydrolysis of the sn-2 ester bond of glycerophospholipids to release lysophosphatidylcholine (lysoPC) and oxidized nonesterified fatty acid $(15,16)$. In humans, Lp-PLA2 circulates in its active form as a complex with low-density lipoprotein (LDL) and high-density lipoprotein (17). After LDL oxidation by reactive oxygen radicals, Lp-PLA2/ ox-LDL complexes are recognized by phospholipase A2 receptor (PLA2R) on the macrophage membrane, and enter the cells to produce free fatty acid and lysophosphatide, while simultaneously promoting macrophage M1 polarization and M1 macrophage release of inflammatory factors like tumor necrosis factor (TNF)- $\alpha$ and interleukin (IL)-6 (18-21). Under ox-LDL-induced oxidative stress, Lp-PLA2 silencing was shown to protect against oxidative stress and cell apoptosis in macrophages (22). In lipopolysaccharide (LPS)-injected rats, hepatic exposure to endotoxin led to rapid increases in biliary and plasma LpPLA2 levels (23). Furthermore, plasma Lp-PLA2 activity was strongly associated with liver damage in our previous study (24).

The above research suggests that Lp-PLA2 activity may have important roles in macrophage polarization, oxidative stress, and inflammatory mediator release. MOJ is also correlated with these factors. Therefore, we speculate that Lp-PLA2 may be related to the injury caused by MOJ. The present study aimed to investigate the associations of dynamic changes in Lp-PLA2 activity and laboratory parameters in patients with MOJ before and after treatment.

We present the following article in accordance with the STROBE Statement reporting checklist (available at http:// dx.doi.org/10.21037/apm-20-133).

\section{Methods}

\section{Patients}

Twenty-one MOJ patients who attended the Department of Hepatobiliary and Pancreatic Surgery, The First Affiliated Hospital, School of Medicine, Zhejiang University, China, between August 2016 and November 2017 were enrolled in this prospective study. The patients comprised 15 males and 6 females aged $44-87$ years (mean age: $66.1 \pm 11.4$ years). Of the 21 patients, 1 was diagnosed with colorectal cancer with hepatic metastasis, 8 with cholangiocarcinoma, 6 with pancreatic cancer, 4 with duodenal cancer, 1 with carcinoma of ampulla, and 1 with cholecystolithiasis. Regarding treatments, 15 (71.4\%) underwent surgical resection of local lesion, $2(9.5 \%)$ underwent drainage by PTCD, 2 (9.5\%) underwent stent insertion by PTCD, 1 (4.8\%) underwent bile duct stone extraction by ERCP, and 1 (4.8\%) underwent ENBD by ERCP. Before and after the surgical or interventional operation, blood samples were collected at specific time points. The study was conducted in accordance with the Declaration of Helsinki (as revised in 2013) and the participants gave informed consent before taking part. The study was approved by the Ethics Committee of The First Affiliated Hospital of Zhejiang University (Approval No. 2017-082).

\section{Biochemical indicator assays}

Blood concentrations of the biochemical indicators were 
measured just before the operation, and at 1 day, 1 week, and day of hospital discharge after the surgical or interventional operation. All specimens for blood indicators and Lp-PLA2 activity measurements were collected by venipuncture into 5 -mL drying Vacuette vacutainers (Greiner Bio-One GmbH, Kremsmunster, Austria) in the morning after a 12 -h fast on day 2 after admission. After the samples were sent to the laboratory, serum was isolated by centrifugation $(10 \mathrm{~min}, 3,000 \times \mathrm{g})$ and preserved at $-80{ }^{\circ} \mathrm{C}$ until analysis. Lp-PLA2 activity, total protein (TP), albumin (ALB), alkaline phosphatase (ALP), alanine aminotransferase (ALT), aspartate aminotransferase (AST), gamma-glutamyltransferase (GGT), total bilirubin (TB), direct bilirubin (DB), total bile acid (TBA), amylase (Amy), and high-sensitivity C-reactive protein (Hs-CRP) levels were measured with a Hitachi 7600 automatic biochemical analyzer (Hitachi, Tokyo, Japan) using Roche reagents (Roche Diagnostics, Mannheim, Germany) for TP, ALB, ALT, AST, GGT, TB, and DB, Shengsuoyoufu reagents (SSUF, Shanghai, China) for Hs-CRP, TBA, and Amy, and an Auto LP-PLA2 Kit (Diasys, Holzheim, Germany) for Lp-PLA2 activity measurements. All biochemical parameters were conducted in the same laboratory and the laboratory quality control is within the control range.

\section{Statistical analysis}

Statistical analyses were performed using SPSS version 22.0 software (SPSS Inc., Chicago, IL, USA). Data were expressed as mean and standard deviation for continuous variables, and as median and range for categorical variables. Spearman rank correlation analysis was used to evaluate the relationships between Lp-PLA2 activity and other parameters. The statistical significance of differences in postoperative data at 1 day, 1 week, and day of hospital discharge relative to the preoperative baseline values was evaluated by repeated-measures analysis of variance. To examine the differences in Lp-PLA2 activity and other parameters, percent differences were calculated by the following equation: (postoperative value - preoperative baseline value) $\times 100 \% /$ baseline value. Box plots were created to demonstrate the Lp-PLA2 activity and levels of enzyme indicators. Dynamic difference plots of the percent changes in Lp-PLA2 activity and other parameters were drawn with the percent changes on the $\mathrm{Y}$-axis and the postoperative time points on the $\mathrm{X}$-axis. Scatter plots for the four Lp-PLA2 activity measurements in each patient were created, with $0-21$ representing the 21 patients on the $\mathrm{X}$-axis, and the Lp-PLA2 activity levels on the $\mathrm{Y}$-axis. Values of $\mathrm{P}<0.05$ were considered statistically significant.

\section{Results}

\section{Clinical characteristics of the patients at specific time points}

The levels of TP, ALB, ALP, ALT, AST, GGT, TB, DB, TBA, Amy, Lp-PLA2 activity, and Hs-CRP before the operation, and at 1 day, 1 week, and day of hospital discharge after the operation are shown in Table 1. The levels of TP, ALB, ALP, ALT, AST, GGT, TB, DB, TBA, Lp-PLA2 activity, and Hs-CRP differed significantly among the four paired time points $(\mathrm{P}<0.05$ for all). The levels of ALP, AST, GGT, TB, DB, TBA, and Lp-PLA2 activity at 1 day, 1 week, and day of hospital discharge after the operation differed significantly from the baseline levels before the operation $(\mathrm{P}<0.05$ for all). The levels of $\mathrm{TB}$, DB, and Lp-PLA2 activity at 1 week and day of hospital discharge after the operation differed significantly from the values at 1 day after the operation $(\mathrm{P}<0.05$ for all $)$. The levels of ALB, ALP, ALT, AST, GGT, TB, DB, and HsCRP at day of hospital discharge differed significantly from the values at 1 week postoperatively $(\mathrm{P}<0.05$ for all). As shown in Figure 1, the levels of enzyme indicators (ALP, ALT, AST, GGT, Lp-PLA2), bilirubin indicators (TB, DB), and TBA gradually decreased, while the Hs-CRP level initially increased and then decreased.

\section{Correlation analyses between Lp-PLA2 activity and laboratory parameters}

Spearman correlation analysis was employed to determine the correlations between Lp-PLA2 activity and laboratory parameters at the preoperative and postoperative time points. The results revealed that Lp-PLA2 activity at baseline was significantly positively correlated with $\mathrm{TB}$ $(\mathrm{r}=0.463, \mathrm{P}=0.035)$, but not correlated with TP, ALB, ALP, ALT, AST, GGT, DB, TBA, Amy, and Hs-CRP $(\mathrm{P}>0.05$ for all). Lp-PLA2 activity at 1 day postoperatively was significantly negatively correlated with Hs-CRP ( $r=-0.600$, $\mathrm{P}=0.004)$, positively correlated with $\mathrm{TB}(\mathrm{r}=0.558, \mathrm{P}=0.009)$ and $\mathrm{DB}(\mathrm{r}=0.509, \mathrm{P}=0.019)$, and not correlated with other parameters $(\mathrm{P}>0.05$ for all). Lp-PLA2 activity at 1 week postoperatively was significantly negatively correlated with Hs-CRP ( $\mathrm{r}=-0.605, \mathrm{P}=0.005)$ and positively correlated with TB $(r=0.559, P=0.008)$ and $\mathrm{DB}(\mathrm{r}=0.455, \mathrm{P}=0.038)$. Finally, 
Table 1 Clinical characteristics of the patients at specific time points

\begin{tabular}{|c|c|c|c|c|c|}
\hline Parameters & Before the operation & 1 day & 1 week & Day of hospital discharge & $\mathrm{P}$ \\
\hline ALB (g/L) & $34.7 \pm 5.5$ & $31.3 \pm 4.5^{\star}$ & $33.8 \pm 4.4$ & $37.0 \pm 4.33^{\# \&}$ & 0.002 \\
\hline ALP (U/L) & $411[216-1,532]$ & $196[112-1,017]^{*}$ & $205[124-706]^{*}$ & $125[61-318]^{\# \#}$ & $<0.001$ \\
\hline ALT (U/L) & 83 [17-468] & $77[24-416]^{\star}$ & $51[11-403]$ & $26[9-149]^{{ }^{\# \&}}$ & $<0.001$ \\
\hline GGT (U/L) & $627[33-1,530]$ & $170[49-682]^{*}$ & $134[33-703]^{*}$ & $76[23-204]^{]^{\# \&}}$ & $<0.001$ \\
\hline TB $(\mu \mathrm{mol} / \mathrm{L})$ & $220[72-477]$ & $151[40-412]^{\star}$ & $70[14-418]^{\star^{\#}}$ & $19[6-709]^{]^{\# \&}}$ & $<0.001$ \\
\hline $\mathrm{DB}(\mu \mathrm{mol} / \mathrm{L})$ & $177[44-374]$ & $101[30-332]^{*}$ & $47[9-309]^{\star^{\#}}$ & $9[3-519]^{\star^{\# \&}}$ & $<0.001$ \\
\hline TBA $(\mu \mathrm{mol} / \mathrm{L})$ & $130[4-264]$ & $7[3-224]^{*}$ & $9[2-70]^{*}$ & $9[6-103]^{\star}$ & 0.001 \\
\hline LP-PLA2 (U/L) & $636[172-1,664]$ & $646[175-1,025]^{*}$ & $419[144-949]^{\star^{*}}$ & $347[165-698]^{]^{\#}}$ & $<0.001$ \\
\hline
\end{tabular}

*, the values at 1 day, 1 week, or day of hospital discharge after the operation were compared with the baseline values before the operation. ", the values at 1 week or day of hospital discharge after the operation were compared with values at 1 day after the operation. \& the values at day of hospital discharge were compared with the values at 1 week after the operation. TP, total protein; ALB, albumin; ALP, alkaline phosphatase; ALT, alanine aminotransferase; AST, aspartate aminotransferase; GGT, gamma-glutamyltransferase; TB, total bilirubin; DB, direct bilirubin; TBA, total bile acid; Amy, amylase; CRP, C-reactive protein.

Lp-PLA2 activity at day of hospital discharge was not correlated with other parameters ( $\mathrm{P}>0.05$ for all). Thus, we found that Lp-PLA2 activity was correlated with TB, DB, and Hs-CRP at specific time points. To further examine the correlations, we calculated the difference rates and drew dynamic difference graphs to show the dynamic changes.

\section{Dynamic changes in LP-PLA2 activity and relevant parameters at specific preoperative and postoperative time points}

The dynamic changes in the three difference rates for the parameters are shown in Table 2. D1, D2, and D3 for LpPLA2, ALP, ALT, AST, GGT, TB, DB, and TBA gradually decreased, meaning that the levels of these indicators gradually decreased after biliary obstruction was relieved by surgical treatment. D1 and D2 of Hs-CRP were both increased, while D3 decreased. The dynamic difference graphs for ALP, ALT, AST, GGT, TB, DB, TBA, and LpPLA2 are shown in Figure 2. The visual dynamic changes in these parameters and the respective changes at different points also showed that these parameters gradually decreased after biliary obstruction was relieved by surgical or interventional treatment.

\section{Associations of clinical outcomes and Lp-PLA2 activity}

After pancreatoduodenectomy or minimally invasive treatment combined with medication (mainly ursodeoxycholic acid), the biliary obstruction in the 21 patients was alleviated and they were discharged from the hospital. The LpPLA2 activity measurements for the individual patients are shown in Figure 3. The data clearly showed that the fourth Lp-PLA2 activity measurements in most patients were $<600 \mathrm{U} / \mathrm{L}$ [close to the upper limit of the normal reference range (25)] and lower than the previous three measurements.

\section{Discussion}

In the present study, we investigated the associations between serum Lp-PLA2 activity dynamic changes and biliary obstruction in MOJ patients. Lp-PLA2 activity was associated with TB at all time points, and the difference rates and dynamic difference graphs showed that postoperative Lp-PLA2, ALP, ALT, AST, GGT, TB, DB, and TBA gradually decreased after biliary obstruction was relieved by surgical or interventional treatment.

The main pathological factor caused by MOJ is 

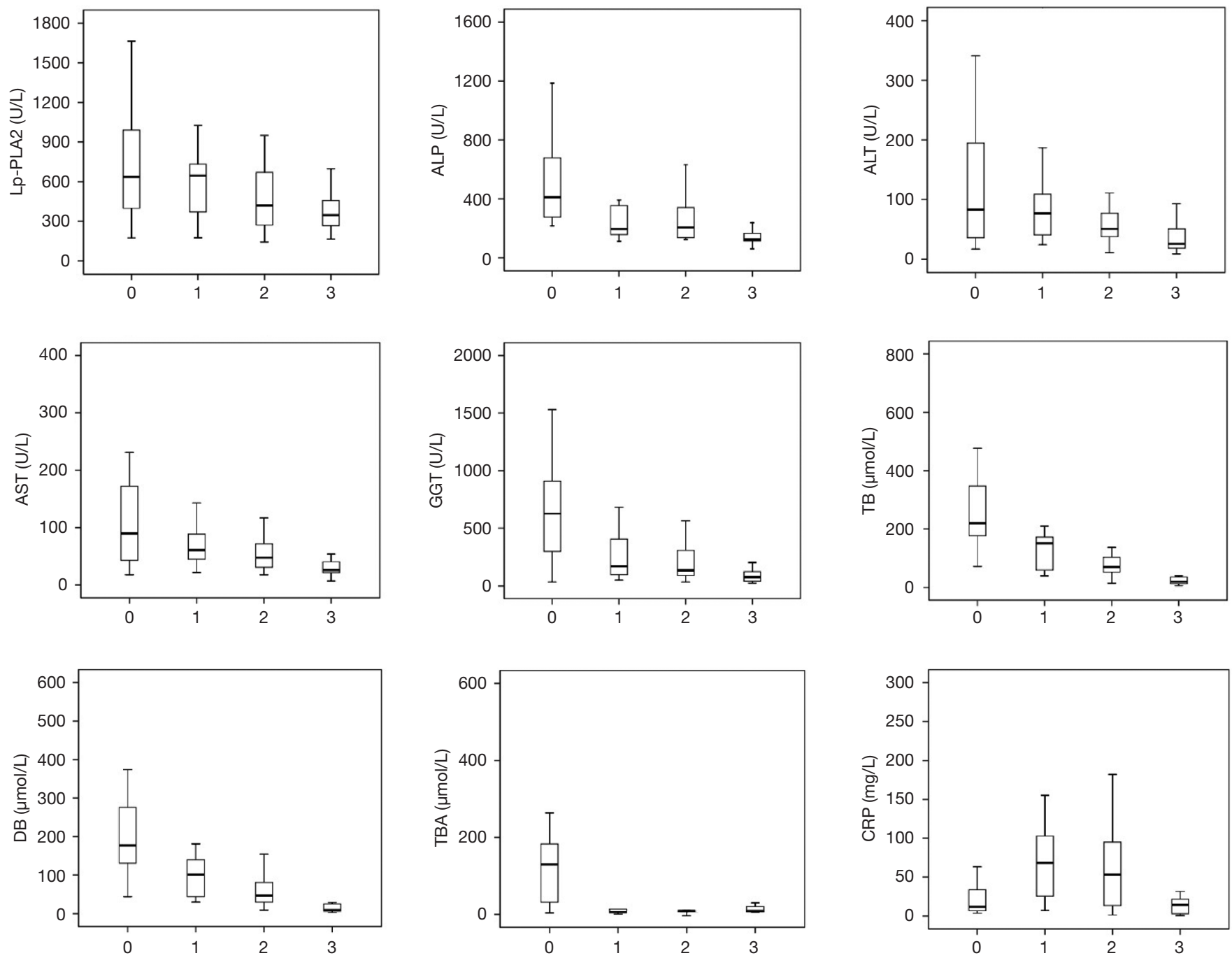

Figure 1 Box plots of Lp-PLA2 activity and levels of enzyme indicators at the examined time points. Y-axis: Lp-PLA2 activity, and ALP, ALT, AST, GGT, TB, DB, TBA, and CRP levels. X-axis: 0, preoperative; 1, postoperative 1 day; 2, postoperative 1 week; 3, day of hospital discharge. ALP, alkaline phosphatase; ALT, alanine aminotransferase; AST, aspartate aminotransferase; GGT, gamma-glutamyltransferase; TB, total bilirubin; DB, direct bilirubin; TBA, total bile acid; CRP, C-reactive protein.

enterogenous endotoxemia (6). Endotoxin is a type of LPS, a cell wall component of Gram-negative bacteria (26). In MOJ, the lack of bile salt, impaired intestinal mucosal barrier, and disrupted intestinal immune barrier function result in absorption of endotoxin into the liver through the portal vein $(6,27,28)$. In addition, the activity of liver Kupffer cells (KCs) is inhibited, and their functions in phagocytosis and cell killing are impaired, meaning that KCs cannot effectively remove endotoxin $(29,30)$. Consequently, endotoxemia occurs, and the invading endotoxin activates $\mathrm{KCs}$ to induce their secretion of various inflammatory factors, such as TNF- $\alpha$, IL-6, and Lp-PLA2, that mediate inflammatory reactions and cause body damage (31-33). Furthermore, the activated KCs release large amounts of oxygen free radicals that can mediate liver damage in MOJ (31). Endotoxin also induces KCs to produce nitric oxide synthase (iNOS), nitric oxide, malondialdehyde (MDA), and reactive oxygen species (ROS) (34-38). Taken together, these findings suggest that KCs mediate liver damage by secreting oxygen free radicals and producing oxidative stress.

LPS stimulation can affect Lp-PLA2 expression. Previous 
Table 2 Difference rates for Lp-PLA2 activity and other parameters

\begin{tabular}{lccc}
\hline Parameters & \multicolumn{1}{c}{ D1 } & D2 & D3 \\
\hline TP $(\mathrm{g} / \mathrm{L})$ & $-0.12(-0.37$ to 0.01$)$ & $-0.11(-0.31$ to 0.1$)$ & $0.02(-0.24$ to 0.47$)$ \\
ALB $(\mathrm{g} / \mathrm{L})$ & $-0.08(-0.35$ to 0.22$)$ & $0.02(-0.32$ to 0.25$)$ & $0.10(-0.22$ to 0.44$)$ \\
ALP $(\mathrm{U} / \mathrm{L})$ & $-0.50(-0.87$ to 0.22$)$ & $-0.54(-0.89$ to 1.45$)$ & $-0.73(-0.90$ to -0.08$)$ \\
ALT $(\mathrm{U} / \mathrm{L})$ & $-0.21(-0.88$ to 10.56$)$ & $-0.56(-0.95$ to 4.43$)$ & $-0.75(-0.97$ to 3.26$)$ \\
AST $(\mathrm{U} / \mathrm{L})$ & $-0.32(-0.81$ to 9.73$)$ & $-0.46(-0.92$ to 4.28$)$ & $-0.69(-0.96$ to 1.41$)$ \\
GGT $(\mathrm{U} / \mathrm{L})$ & $-0.48(-0.91$ to 1.28$)$ & $-0.69(-0.95$ to 3.06$)$ & $-0.84(-0.97$ to 0.86$)$ \\
TB $(\mu \mathrm{mol} / \mathrm{L})$ & $-0.48(-0.82$ to 0.13$)$ & $-0.68(-0.91$ to 0.07$)$ & $-0.91(-0.97$ to 0.81$)$ \\
DB $(\mu \mathrm{mol} / \mathrm{L})$ & $-0.54(-0.81$ to 0.12$)$ & $-0.69(-0.93$ to 0.07$)$ & $-0.94(-0.98$ to 0.80$)$ \\
TBA $(\mu \mathrm{mol} / \mathrm{L})$ & $-0.88(-0.98$ to 0.61$)$ & $-0.92(-0.99$ to 2.68$)$ & $-0.91(-0.98$ to 4.42$)$ \\
Amy $(\mathrm{U} / \mathrm{L})$ & $0.21(-0.79$ to 17.0$)$ & $0.11(-0.96$ to 2.94$)$ & $-0.17(-0.95$ to 3.57$)$ \\
CRP $(\mathrm{mg} / \mathrm{L})$ & $1.84(-0.28$ to 21.55$)$ & $1.64(-0.85$ to 13.89$)$ & $-0.45(-0.99$ to 15.32$)$ \\
LP-PLA2 $(\mathrm{U} / \mathrm{L})$ & $-0.25(-0.56$ to 1.07$)$ & $-0.32(-0.74$ to 0.5$)$ & $-0.45(-0.86$ to 0.94$)$ \\
\hline
\end{tabular}

Three kinds of difference rates were examined: D1 = (value at 1 day postoperatively - baseline)/baseline; D2 = (value at 1 week postoperatively - baseline)/baseline; D3 = (value at day of hospital discharge - baseline)/baseline. TP, total protein; ALB, albumin; ALP, alkaline phosphatase; ALT, alanine aminotransferase; AST, aspartate aminotransferase; GGT, gamma-glutamyltransferase; TB, total bilirubin; DB, direct bilirubin; TBA, total bile acid; Amy, amylase; CRP, C-reactive protein.

studies showed that Lp-PLA2 expression was increased in a murine macrophage cell line and human THP-1 cells through the mitogen-activated protein pathway after LPS exposure $(39,40)$. Howard et al. (19) reported a 2 -fold increase in plasma Lp-PLA2 activity after LPS exposure for 24 hours in a rat model. Svetlov et al. (41) described a maximal 4-5-fold increase in bile Lp-PLA2 activity after LPS infusion for 2.5 hours, and found that plasma LpPLA2 activity was significantly elevated after LPS exposure for 18 hours. Sun et al. (42) demonstrated that LPS, TNF- $\alpha$, and ROS levels stimulated an increase in Lp-PLA2 expression. Furthermore, the relationship between LPS and Lp-PLA2 was closely related to KCs. Howard et al. (19) confirmed that the rat liver responds to LPS exposure with increased Lp-PLA2 mRNA and protein expression mainly in the resident KCs in the liver, rather than hepatocytes or endothelial cells, and that the secretion rate of Lp-PLA2 by KCs was 20-25 times higher than that by hepatocytes. LPS can activate Toll-like receptor 4 (TLR4) and CD14 on the KCs membrane $(23,43)$. KCs subsequently secrete Lp-PLA2 into the peripheral blood or surrounding tissue, where it releases lysoPC and fatty acid $(20,44)$. In turn, lysoPC increases certain inflammatory cytokine (IL-1 $\beta$, IL-6, TNF- $\alpha$ ) levels in a concentration-dependent manner $(20,44)$. Meanwhile, Shi et al. (44) found Lp-PLA2 activity mediated leukocyte activation and inflammatory responses in an in vitro cell model. Because PLA2R is expressed on KCs (19-21), we speculate that Lp-PLA2 enters KCs through this receptor to mediate leukocyte activation and inflammatory responses and promote macrophage inflammatory M1 polarization, leading to M1 macrophage release of inflammatory factors like TNF- $\alpha$ and IL-6 (44-46).

Other studies found that Lp-PLA2 was closely related to oxidative stress in KCs $(42,47)$. Oxidative stress plays an important role in the development of diseases (42). Some medications against oxidative stress and Lp-PLA2 inhibitors inhibited KCs activation and reduced the inflammatory process, thereby reducing liver damage and enhancing liver recovery $(42,47,48)$. Resveratrol inhibited Lp-PLA2 expression in macrophages and this inhibition was associated with reductions in inflammation and ROS levels (42). Simvastatin inhibited oxidative stress by improving SOD functional activity, increasing GSH-Px and HO-1, and decreasing MDA generation (47). Furthermore, simvastatin treatment significantly down-regulated LPS-induced LpPLA2 (released by macrophages) mRNA and protein expression, and inhibited Lp-PLA2 activity in a dose- and time-dependent manner $(39,40)$. darapladib is an Lp-PLA2specific inhibitor that decreased iNOS and intra-cellular adhesion molecule (ICAM)-1 expression in a rat model (48). 

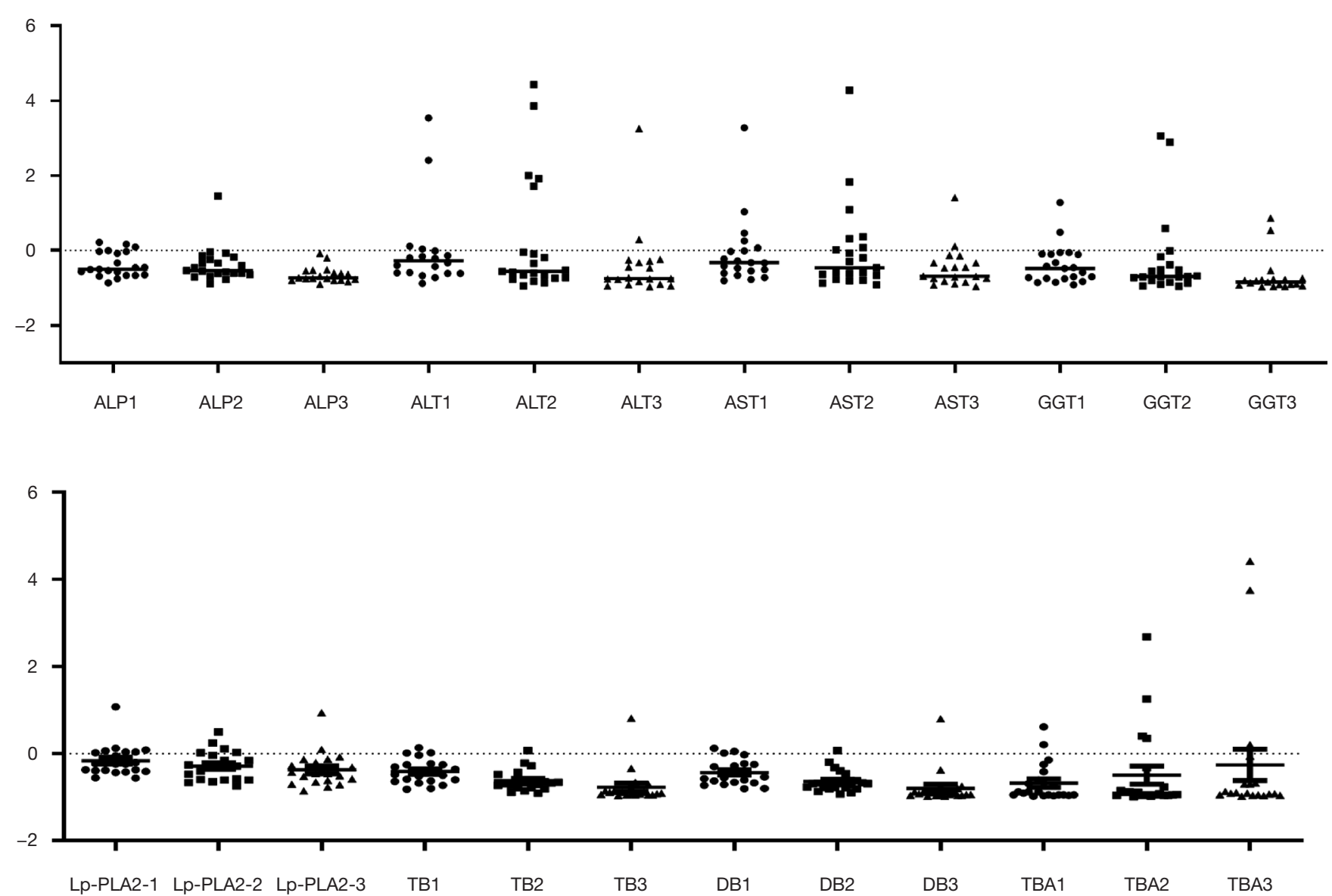

Figure 2 Dynamic difference graphs for Lp-PLA2 and biochemical indicators in 21 patients after operation. Lp-PLA2-1, ALP1, ALT1, AST1, GGT1, TB1, BD1, and TBA1: percentage changes at postoperative 1 day (D1). Lp-PLA2-2, ALP2, ALT2, AST2, GGT2, TB2, BD2, and TBA2: percentage changes at postoperative 1 week (D2). Lp-PLA2-3, ALP3, ALT3, AST3, GGT3, TB3, BD3, and TBA3: percentage changes at day of hospital discharge (D3). ALP, alkaline phosphatase; ALT, alanine aminotransferase; AST, aspartate aminotransferase; GGT, gamma-glutamyltransferase; TB, total bilirubin; DB, direct bilirubin; TBA, total bile acid.

Zheng et al. (22) reported that Lp-PLA2 silencing protected against oxidative stress in human macrophages by downregulating ROS and MDA and increasing SOD.

Above all, Lp-PLA2 activity may be associated with biliary obstruction in MOJ patients, mainly through $\mathrm{KC}$-mediated enterogenous endotoxemia and oxidative stress. In this study, we found that ALP, ALT, AST, GGT, TB, DB, TBA, and Lp-PLA2 activity were increased in MOJ patients preoperatively, and that Lp-PLA2 activity was correlated with TB and DB levels, reflecting liver damage. Similarly, our previous study showed that LpPLA2 activity was significantly associated with TB in HBV patients with liver damage (24). Svetlov et al. (49) proposed that increased biliary and plasma Lp-PLA2 activity was involved in the hepatic response to endotoxic exposure in a rat model. It is clear that Lp-PLA2 activity can act as an indicator of MOJ-induced liver damage. We also found that Lp-PLA2 activity, ALP, ALT, AST, GGT, TB, DB, and TBA gradually decreased after biliary obstruction was relieved by surgical or interventional treatment, and were lowest at day of hospital discharge, which also indicated improvement in liver functions. This relationship between Lp-PLA2 activity and liver function was also observed in the following studies. Meade et al. (50) found that patients with chronic cholestasis, especially stage III or IV primary or secondary biliary cirrhosis or cholangiocarcinoma, exhibited increased serum Lp-PLA2 activity, while LpPLA2 activity was reduced to normal or near-normal levels after liver transplantation with recovery of liver function. Thus, it is probable that the liver has an important role 


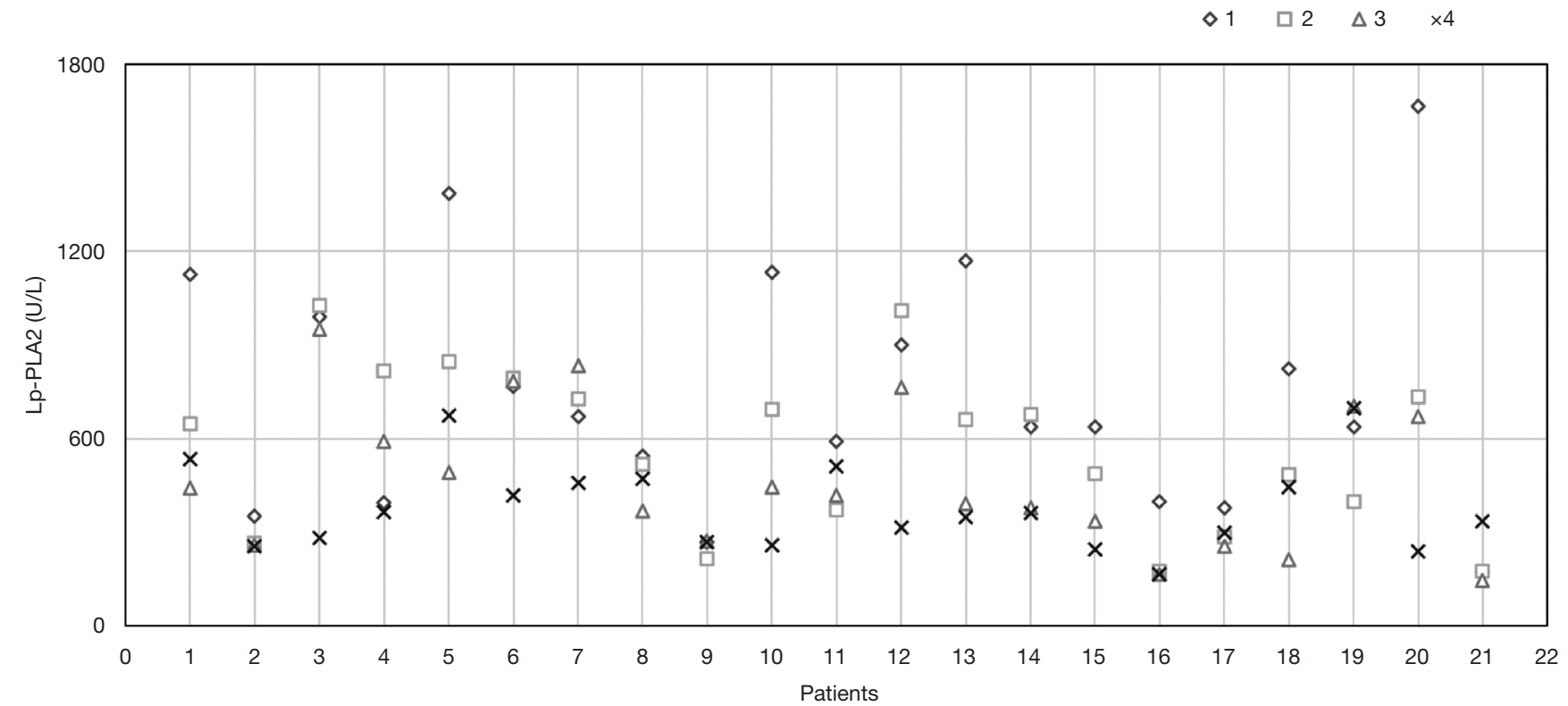

Figure 3 Scatter plots of Lp-PLA2 activity measurements for individual patients at the examined time points. Numbers 1 to 21 represent the 21 patients. $\diamond$, preoperative Lp-PLA2 activity; $\square$, Lp-PLA2 activity at postoperative 1 day; $\triangle$, Lp-PLA2 activity at postoperative 1 week; $\times$, Lp-PLA2 activity at day of hospital discharge.

in the regulation of serum Lp-PLA2 activity. Kamisako et al. (51) found that serum Lp-PLA2 activity increased in hyperbilirubinemia associated with liver cirrhosis, obstructive cholestasis, or acute hepatitis, and that LpPLA2 activity decreased after remission in acute hepatitis and treatment in obstructive cholestasis. Thus, we speculate that LPS stimulates Lp-PLA2 to enter KCs in the liver, mediates leukocyte activation and inflammatory responses, and causes M1 macrophages to release inflammatory factors. After surgery or interventional treatment leading to obstruction relief and endotoxemia reduction, the liver inflammatory responses and liver injury begin to recover and KCs reduce their secretion of Lp-PLA2, leading to a gradual reduction in serum Lp-PLA2. By monitoring the dynamic changes in Lp-PLA2 activity in MOJ patients, we can observe the effects of surgery or interventional treatment in MOJ patients.

In summary, our findings revealed the dynamic changes in Lp-PLA2 activity in MOJ patients, and demonstrated that Lp-PLA2 activity was associated with biliary obstruction and liver damage and can be used as a novel indicator for severity of biliary obstruction and treatment monitoring. However, the small sample size is a limitation of the present study, and further studies are warranted to confirm our findings.

\section{Acknowledgments}

The authors thank Alison Sherwin, PhD, from Liwen Bianji, Edanz Group China (www.liwenbianji.cn/ac) for editing the English text of a draft of this manuscript.

Funding: This work was supported by grants from the Science and Technology Project (LGF19H040017), Natural Science Foundation (LY20H200005), and Education Department (Y201636519) of Zhejiang Province.

\section{Footnote}

Reporting Checklist: The authors have completed the STROBE reporting checklist. Available at http://dx.doi. org/10.21037/apm-20-133

Data Sharing Statement: Available at http://dx.doi. org/10.21037/apm-20-133

Conflicts of Interest: All authors have completed the ICMJE uniform disclosure form (available at http://dx.doi. org/10.21037/apm-20-133). The authors have no conflicts of interest to declare.

Ethical Statement: The authors are accountable for all 
aspects of the work in ensuring that questions related to the accuracy or integrity of any part of the work are appropriately investigated and resolved. The study was conducted in accordance with the Declaration of Helsinki (as revised in 2013) and the participants gave informed consent before taking part. The study was approved by the Ethics Committee of The First Affiliated Hospital of Zhejiang University (Approval No. 2017-082).

Open Access Statement: This is an Open Access article distributed in accordance with the Creative Commons Attribution-NonCommercial-NoDerivs 4.0 International License (CC BY-NC-ND 4.0), which permits the noncommercial replication and distribution of the article with the strict proviso that no changes or edits are made and the original work is properly cited (including links to both the formal publication through the relevant DOI and the license). See: https://creativecommons.org/licenses/by-nc-nd/4.0/.

\section{References}

1. Fargo MV, Grogan SP, Saguil A. Evaluation of Jaundice in Adults. Am Fam Physician 2017;95:164-8.

2. Shen $Z$, Tian L, Wang X. Treatment of pancreatic head cancer with obstructive jaundice by endoscopy ultrasonography-guided gastrojejunostomy: A case report and literature review. Medicine (Baltimore) 2018;97:e11476.

3. Dasari BVM, Ionescu MI, Pawlik TM, et al. Outcomes of surgical resection of gallbladder cancer in patients presenting with jaundice: A systematic review and metaanalysis. J Surg Oncol 2018;118:477-85.

4. Bertani H, Frazzoni M, Mangiafico S, et al. Cholangiocarcinoma and malignant bile duct obstruction: A review of last decades advances in therapeutic endoscopy. World J Gastrointest Endosc 2015;7:582-92.

5. Gholami S, Brennan MF. Preoperative Stenting for Benign and Malignant Periampullary Diseases: Unnecessary if Not Harmful. Surg Clin North Am 2018;98:37-47.

6. Pavlidis ET, Pavlidis TE. Pathophysiological consequences of obstructive jaundice and perioperative management. Hepatobiliary Pancreat Dis Int 2018;17:17-21.

7. Modha K. Clinical Approach to Patients With Obstructive Jaundice. Tech Vasc Interv Radiol 2015;18:197-200.

8. Farrukh SZ, Siddiqui AR, Haqqi SA, et al. Comparison Of Ultrasound Evaluation Of Patients Of Obstructive Jaundice With Endoscopic Retrograde CholangioPancreatography Findings. J Ayub Med Coll Abbottabad
2016;28:650-2.

9. Antonini F, Macarri G. Endoscopic ultrasound for the diagnosis of an uncommon cause of obstructive jaundice masquerading as malignancy. Endosc Ultrasound 2017;6:276-7.

10. Singh A, Mann HS, Thukral CL, et al. Diagnostic Accuracy of MRCP as Compared to Ultrasound/CT in Patients with Obstructive Jaundice. J Clin Diagn Res 2014;8:103-7.

11. Shanmugarajah I, Solhaug M, Aslam O, et al. Efficacy and safety assessment of ERCP in patients with malignant biliary obstruction. Acta Gastroenterol Belg 2017;80:487-91.

12. Tang K, Sui LL, Xu G, et al. Effects of Different Palliative Jaundice Reducing Methods on Immunologic Functions in Patients with Advanced Malignant Obstructive Jaundice. Anticancer Res 2017;37:4665-70.

13. Choi J, Ryu JK, Lee SH, et al. Biliary drainage for obstructive jaundice caused by unresectable hepatocellular carcinoma: the endoscopic versus percutaneous approach. Hepatobiliary Pancreat Dis Int 2012;11:636-42.

14. Sikora SS, Kapoor R, Pradeep R, et al. Palliative surgical treatment of malignant obstructive jaundice. Eur J Surg Oncol 1994;20:580-4.

15. Karabina SA, Gora S, Atout R, et al. Extracellular phospholipases in atherosclerosis. Biochimie 2010;92:594-600

16. Tjoelker LW, Stafforini DM. Platelet-activating factor acetylhydrolases in health and disease. Biochim Biophys Acta 2000;1488:102-23.

17. Tellis CC, Tselepis AD. Pathophysiological role and clinical significance of lipoprotein-associatedphospholipase A2 (Lp-PLA2) bound to LDL and HDL. Curr Pharm Des 2014;20:6256-69.

18. Peplow PV. Regulation of platelet-activating factor (PAF) activity in human diseases by phospholipase $\mathrm{A} 2$ inhibitors, PAF acetylhydrolases, PAF receptor antagonists and free radical scavengers. Prostaglandins Leukot Essent Fatty Acids 1999;61:65-82.

19. Howard KM, Abdel-AM, Ditmyer M, et al. Lipopolysaccharide and platelet-activating factor stimulate expression of platelet-activating factor acetylhydrolase via distinct signaling pathways. Inflamm Res 2011;60:735-44.

20. Morioka Y, Saiga A, Yokota Y, et al. Mouse group X secretory phospholipase $\mathrm{A} 2$ induces a potent release of arachidonic acid from spleen cells and acts as a ligand for the phospholipase A2 receptor. Arch Biochem Biophys 2000;381:31-42. 
21. Heriansyah T, Nafisatuzzamrudah N, Aini FN, et al. Reduction in Vasa Vasorum Angiogenesis by LpPLA2 Selective Inhibitor Through The HIF-1 $\alpha$ and VEGF Expression Under Dyslipidemic Conditions in Atherosclerosis Pathogenesis. Cardiovasc Hematol Agents Med Chem 2018;16:114-9.

22. Zheng H, Cui D, Quan X, et al. Lp-PLA2 silencing protects against ox-LDL-induced oxidative stress and cellapoptosis via Akt/mTOR signaling pathway in human THP1 macrophages. Biochem Biophys Res Commun 2016;477:1017-23.

23. Wang ZK, Xiao JG, Huang XF, et al. Effect of biliary drainage on inducible nitric oxide synthase, CD14 and TGR5expression in obstructive jaundice rats. World J Gastroenterol 2013;19:2319-30.

24. Feng L, Zhao Y, Feng G, et al. Clinical application of elevated platelet-activating factor acetylhydrolase in patients with hepatitis B. Lipids Health Dis 2014;13:105.

25. Feng LM, Feng GF, Chen Y. Evaluation of lipoproteinassociated phospholipase A2 in healthy Chinese Han adult serum. Lipids Health Dis 2014;13:6.

26. Maldonado RF, Sá-Correia I, Valvano MA. Lipopolysaccharide modification in Gram-negative bacteria during chronic infection. FEMS Microbiol Rev 2016;40:480-93.

27. Parlesak A, Schaeckeler S, Moser L, et al. Conjugated primary bile salts reduce permeability of endotoxin through intestinal epithelial cells and synergize with phosphatidylcholine in suppression of inflammatory cytokine production. Crit Care Med 2007;35:2367-74.

28. Ota S, Suzuki S, Sakaguchi T, et al. Significance of morphological alteration by portal vein branch ligation in endotoxin-induced liver injury after partial hepatectomy. Liver Int 2007;27:1076-85.

29. Li W, Chan AC, Lau JY, et al. Superoxide and nitric oxide production by Kupffer cells in rats with obstructive jaundice: effect of internal and external drainage. J Gastroenterol Hepatol 2004;19:160-5.

30. Abrahám S, Hermesz E, Szabó A, et al. Effects of Kupffer cell blockade on the hepatic expression of metallothionein and heme oxygenase genes in endotoxemic rats with obstructive jaundice. Life Sci 2012;90:140-6.

31. Meng Y, Gong YC, Dou Y, et al. Changes of serum cytokines and expression of inducible nitric oxide synthase mRNA by Kupffer cells after relief from obstructive jaundice in rats. J Gastroenterol Hepatol 2009;24:1064-9.

32. Howard KM, Miller JE, Miwa M, et al. Cell-specific regulation of expression of plasma-type platelet- activating factor acetylhydrolase in the liver. J Biol Chem 1997;272:27543-8.

33. Howard KM, Olson MS. The expression and localization of plasma platelet-activating factor acetylhydrolase in endotoxemic rats. J Biol Chem 2000;275:19891-6.

34. Beyazit Y, Sayilir A, Koklu S, et al. Serum nitric oxide levels in patients with benign and malignant biliary disease: a prospective single center study. J Clin Gastroenterol 2013;47:258-63.

35. Liao MH, Shih CC, Tsao CM, et al. RhoA/Rhokinase and nitric oxide in vascular reactivity in rats with endotoxaemia. PLoS One 2013;8:e56331.

36. Teixeira-da-Cunha MG, Gomes RN, Roehrs N, et al. Bacterial clearance is improved in septic mice by plateletactivating factor-acetylhydrolase (PAF-AH) administration. PLoS One 2013;8:e74567.

37. Latchoumycandane C, Marathe GK, Zhang R, et al. Oxidatively truncated phospholipids are required agents of tumor necrosis factor $\alpha(\mathrm{TNF} \alpha)$-induced apoptosis. J Biol Chem 2012;287:17693-705.

38. Grypioti AD, Kostopanagiotou G, Mykoniatis M. Plateletactivating factor inactivator (rPAF-AH) enhances liver's recovery after paracetamol intoxication. Dig Dis Sci 2007;52:2580-90.

39. Song JX, Ren JY, Chen H. Simvastatin reduces lipoproteinassociated phospholipase A2 in lipopolysaccharidestimulated human monocyte-derived macrophages through inhibition of the mevalonate-geranylgeranyl pyrophosphate-RhoA-p38 mitogen-activated protein kinase pathway. J Cardiovasc Pharmacol 2011;57:213-22.

40. Jin WY, Ren JY, Chen H. Simvastatin suppress lipopolysaccharides induced upregulation of lipoprotein associated phospholipase A(2) expression in macrophages via inactivation of $\mathrm{p} 38 \mathrm{MAPK}$ pathway. Zhonghua Xin Xue Guan Bing Za Zhi 2010;38:923-8.

41. Svetlov SI, Howard KM, Miwa M, et al. Interaction of platelet-activating factor with rat hepatocytes: uptake, translocation, metabolism, and effects on PAF-acetylhydrolase secretion and protein tyrosine phosphorylation. Arch Biochem Biophys 1996;327:113-22.

42. Sun S, Zhang M, Yang Q, et al. Resveratrol Suppresses Lipoprotein-Associated Phospholipase A 2 Expression by Reducing Oxidative Stress in Macrophages and Animal Models. Mol Nutr Food Res 2017. doi: 10.1002/ mnfr.201601112.

43. Zhang Y, Igwe OJ. Lipopolysaccharide (LPS)-mediated priming of toll-like receptor 4 enhances oxidant-induced prostaglandin E2 biosynthesis in primary murine 
macrophages. Int Immunopharmacol 2018;54:226-37.

44. Shi Y, Zhang P, Zhang L, et al. Role of lipoproteinassociated phospholipase A2 in leukocyte activation and inflammatory responses. Atherosclerosis 2007;191:54-62.

45. Ashley JW, Hancock WD, Nelson AJ, et al. Polarization of Macrophages toward M2 Phenotype Is Favored by Reduction in iPLA2 $\beta$ (Group VIA Phospholipase A2). J Biol Chem 2016;291:23268-81.

46. Rubio JM, Rodríguez JP, Gil-de-Gómez L, et al. Group $\mathrm{V}$ secreted phospholipase A2 is upregulated by IL-4 in human macrophages and mediates phagocytosis via hydrolysis of ethanolamine phospholipids. J Immunol 2015;194:3327-39.

47. Zhang G, Li M, Xu Y, et al. Antioxidation Effect of Simvastatin in Aorta and Hippocampus: A Rabbit Model Fed High-Cholesterol Diet. Oxid Med Cell Longev 2016;2016:6929306.

48. Wihastuti TA, Heriansyah T, Hanifa H, et al. Darapladib

Cite this article as: Zhao Y, Feng G, Feng L. Changes in preoperative and postoperative lipoprotein-associated phospholipase A2 activity in patients with malignant obstructive jaundice. Ann Palliat Med 2020;9(5):2699-2709. doi: 10.21037/apm20-133 inhibits atherosclerosis development in type 2 diabetes mellitus Sprague-Dawley rat model. Endocr Regul 2018;52:69-75.

49. Svetlov SI, Sturm E, Olson MS, et al. Hepatic regulation of platelet-activating factor acetylhydrolase and lecithin:cholesterol acyltransferase biliary and plasma output in rats exposed to bacterial lipopolysaccharide. Hepatology 1999;30:128-36.

50. Meade CJ, Birke F, Metcalfe S, et al. Serum PAFacetylhydrolase in severe renal or hepatic disease in man: relationship to circulating levels of PAF and effects of nephrectomy or transplantation. J Lipid Mediat Cell Signal 1994;9:205-15.

51. Kamisako T, Takeuchi K, Ito T, et al. Serum plateletactivating factor acetylhydrolase (PAF-AH) activity in patients with hyperbilirubinemic hepatobiliary disease. Hepatol Res 2003;26:23-7. 